length and a recrystallization of the moving lattice.

A remarkable feature of the experiment is that the lattice disorder does not reappear upon decreasing the driving force (the applied current) back to zero. This shows that the low-temperature, dynamic annealing procedure yields a vortex configuration with lower free energy than the original field-cooling procedure used to prepare the specimens. To understand

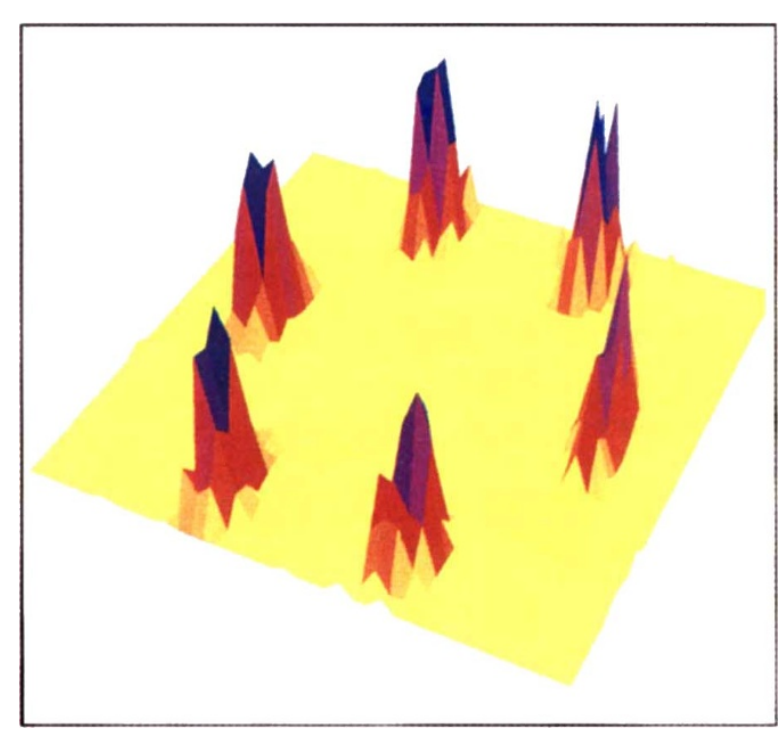

FIG. 2 Scattered neutron intensity on the plane of the detector for a small-angle neutron scattering experiment ${ }^{1}$ in $\mathrm{NbSe}_{2}$ at $1.5 \mathrm{kOe}$ and $4.8 \mathrm{~K}$. The peaks are the six first-order Bragg scattering reflections due to the interaction between the hexagonal magnetic flux-line lattice in the sample and the neutron spin - an effect first predicted by de Gennes and Matricon in 1964. (Courtesy of U. Yaron.)

this, one has to look at the phase diagram of a type II superconductor (see Fig. 1). The arrow marking the 'cooled-in-field' procedure passes through the melting line for the flux lattice. One thus freezes in the disorder existing in the vortex liquid. Once this disorder corresponding to field cooling is removed, it does not come back.

The phenomenon is not restricted to flux lines in a superconductor, but applies to elastic media more generally. The recrystallization effect has recently been predicted by Koshelev and Vinokur ${ }^{2}$, albeit for a two-dimensional system. They show that an elastic medium moving with sufficient speed against random pinning forces experiences a vibrational motion that mimics an annealing process through which disorder that was originally present in the medium is removed. Moreover, their computer simulations also produced the slight increase in disorder just before the onset of uniform flow. In a twodimensional system, this increased disorder shows up as an increased density of edge dislocations.

Duarte et al. from the Centro Atomico in Bariloche, in collaboration with Yaron et al. (manuscript in preparation), very recently produced additional evidence for this model. In their case the structure of the moving vortex lattice was probed at the surface of a single crystal of $\mathrm{NbSe}_{2}$ by magnetic decoration - using fine iron particles to pick out the locations where flux lines leave the crystal - after abruptly switching off the driving current in order to fix the lattice configuration. By repeating the decoration experiment for a series of different driving currents, they were able to demonstrate the disordering of a slowly moving vortex lattice and the recrystallization when it moves quickly enough.

For such phenomena to be observable, it is important that the pinning is weak and fairly uniform. If strong fluctuations in the pinning are assumed, computer simulations made by Jensen et al. $^{3}$ have shown that a different kind of plastic flow occurs. This is usually characterized as partial flow, namely flow of a certain fraction of the vortex lattice through weak-pinning channels which develop amidst strong-pinning islands as the driving force is steadily increased. Such partial flow is believed to be commonly observed in strongpinning, technically useful superconductors and was first described by $\mathrm{Kramer}^{4}$ some 20 years ago.

Its weak pinning and good material properties have made $\mathrm{NbSe}_{2}$ a favourite system for much of the present research in vortex physics. In contrast to the high-critical-temperature superconductors, which have $H_{\mathrm{c} 2}$ of more than 100 tesla at $T=0$, the entire field-temperature diagram is experimentally accessible. Near the melting line, as demonstrated by the noise and transport experiments of Bhattacharya and co-workers ${ }^{5}$, the behaviour of the lattice is plastic flow of the channel kind. The beautiful SANS and transport experiments of Yaron et al. now seem to show us that far below the melting line it is another kind of vortex lattice plasticity, plastic creep, that plays a crucial role in the depinning process at the critical current. $\square$

P. H. Kes is in the Faculty of Mathematics and Natural Sciences, Kamerlingh Onnes Laboratory, University of Leiden, 2300 RA Leiden, The Netherlands.

1. Yaron, U. et al. Nature 376, 753-755 (1995).

2. Koshelev, A. E. \& Vinokur, V. M. Phys. Res. Lett. 73. 3580-3583 (1994).

3. Jensen, H. J., Brass, A. \& Berlinsky, A. J. Phys. Rev. Lett. 60, 1676-1679 (1988)

4. Kramer, E. J. J. appl. Phys. 44, 1360-1370 (1973).

5. Marley, A. C., Higgins, M. J. \& Bhattacharya, S. Phys. Rev. Lett. 74, 3029-3032 (1995)

\section{Chemical skills}

A TRUMPET player once turned up for a concert hopelessly drunk, yet astonished his colleagues by giving a virtuoso performance. "How can you play so well drunk?" they asked him. "Easy: I practise drunk!" he replied. This is 'statedependent learning'. It has been blamed for many examination failures. Students who have learnt their material under the influence of caffeine and nicotine find themselves unable to recall it in the coffee-free, smoke-free examination hall.

At present, the effect is recognized only for addictive drugs. Rats taught a trick under the influence of librium, alcohol or barbiturate may lose the skill when they recover; and conversely. Daedalus cannot see why addictiveness matters. He reckons that many other drugs would show the effect if tested for it. He suspects that nostrums such as garlic, ginseng, kelp, cod-liver oil and so on work this way. Their faithful fans have carelessly allowed some crucial personal skill to come under the control of their nostrum: driving, perhaps, or getting up in the morning, or speaking with apparent authority. Reasonably enough, they feel that they cannot do without it.

Similarly, the many drugs discarded for their adverse effects on rats may merely have exerted state-dependent learning. They may have put the bemused rodents into a state forgetful of some crucial skill of rat life, such as finding the water supply or keeping their nests homelike, thus damaging their health. Alert for such effects, DREADCO rat psychologists are trawling anew the ranks of patent medicines and rejected drugs. Many of them may be safe but powerful agents of state-dependent learning.

The most promising finds will be tested for range and scope on human volunteers. Could a musician practise a dozen different instruments, each under the influence of a specific drug, with each skill unlocked again by its own key? Even the best 'learning drugs' may not be so specific. Even so, some may put the brain into an ideal state for learning music; others mathematics; others social skills or physical dexterity. The user will take the most appropriate pill for learning a given task. To exercise his skill he will simply take another, and then the whole topic will flood back in total recall, with no distraction. Education and adult learning will be transformed. A lecturing pill, a report-writing pill or a committeechairing pill could smooth our climb up the learning curves of these tricky selftaught disciplines. And a passionate-lover pill, if taken regularly during our apprenticeship of this subtle art, should never let us down.

David Jones 